\title{
Unlocking P(V): Reagents for chiral phosphorothioate synthesis
}

\author{
Kyle W. Knouse'*, Justine N. deGruyter"*, Michael A. Schmidt' ${ }^{2}$, Bin Zheng ${ }^{2}$, Julien C. Vantourout ${ }^{1}$, Cian \\ Kingston', Stephen E. Mercer ${ }^{3}$, Ivar M. Medonald ${ }^{3}$, Richard E. Olson ${ }^{3}$, Ye Zhu' ${ }^{2}$ Chao Hang ${ }^{2}$, Jason Zhu', \\ Changxia Yuan ${ }^{2}$, Qinggang Wang ${ }^{2}$, Peter Park ${ }^{4}$, Martin D. Eastgate ${ }^{2}+$, Phil S. Baran' $\uparrow$
}

${ }^{1}$ Department of Chemistry, The Scripps Research Institute, 10550 North Torrey Pines Road, La Jolla, CA 92037, USA. ${ }^{2}$ Chemical and Synthetic Development, Bristol-Myers Squibb, One Squibb Drive, New Brunswick, NJ 08903, USA. ${ }^{3}$ Department of Discovery Chemistry, Bristol-Myers Squibb Research and Development, 5 Research Parkway, Wallingford, CT 06492, USA. ${ }^{4}$ Department of Discovery Chemistry, Bristol-Myers Squibb, P.O. Box 5400, Princeton, NJ 08543, USA.

*These authors contributed equally to this work.

†Corresponding author. Email: michael.schmidt@bms.com (M.A.S.); martin.eastgate@bms.com (M.D.E.); pbaran@scripps.edu (P.S.B.)

Phosphorothioate nucleotides have emerged as powerful pharmacological substitutes of their native phosphodiester analogs with important translational applications in antisense oligonucleotide (ASO) therapeutics and cyclic dinucleotide (CDN) synthesis. Stereocontrolled installation of this chiral motif has long been hampered by the systemic use of $\mathrm{P}(\mathrm{III})$-based reagent systems as the sole practical means of oligonucleotide assembly. A fundamentally different approach is described herein: the invention of a $P(V)$ based reagent platform for programmable, traceless, diastereoselective phosphorus-sulfur incorporation. The power of this reagent system is demonstrated through the robust and stereocontrolled synthesis of various nucleotidic architectures, including ASOs and CDNs, via an efficient, inexpensive, and operationally simple protocol.

Molecular-based gene therapy is rapidly emerging as one of the most promising modes of disease amelioration, as evidenced by the swell of clinical trials employing miRNA, siRNA, and ASOs (1). In a recent example, the ASO SPINRAZA (Fig. 1A) was approved by the FDA as the sole available treatment of spinal muscular atrophy, the leading genetic cause of infant mortality (2). This breakthrough therapy comprises 18 nucleosides bound by phosphorothioate (PS) linkages (i.e., those in which one of the non-bridging oxygen atoms has been substituted for a sulfur). Compared to the native phosphodiester linkage, this simple modification confers a higher degree of metabolic stability and improved cellular uptake through an observation known as the thio effect (3-6). In fact, most FDA-approved ASOs incorporate this moiety, with "numerous" candidates under clinical evaluation (7). Although the PS alteration enhances the pharmacological profile of these systems, it comes at the expense of substantially increased structural complexity-each phosphorus atom is now an uncontrolled stereogenic center (8). Thus, in the case of SPINRAZA, patients receive a mixture of over 100,000 discrete diastereoisomers that, in principle, may bear distinct three-dimensional structures and pharmaceutical properties $(9,10)$. Despite recent studies suggesting that stereodefined systems may improve therapeutic efficacy, readily scalable preparation methods often disregard the stereochemistry at phosphorus, while most of the methods that enable stereocontrol are impractical for large-scale manufacturing (10-
24). As outlined in Fig. 1B, three coupling paradigms have traditionally been employed to facilitate $\mathrm{P}-\mathrm{O}$ bond formation, each relying on cumbersome $\mathrm{P}(\mathrm{III})-$ based reagent strategies. The first (Fig. 1B, i), which produces stereorandom PS-ASOs, commences with a classic phosphoramidite loading step to append the first nucleoside, followed by deprotection, coupling to a second nucleoside, then oxidative sulfurization of the resulting $\mathrm{P}(\mathrm{III})$ species to the $\mathrm{P}(\mathrm{V})$ state $(11,12)$. The second strategy (Fig. 1B, iii), capable of accessing stereodefined PS linkages, uses a phosphoramidate containing a P(III)centered chiral auxiliary to control the phosphorous stereochemistry, but is otherwise identical to the first. The most mature manifestation of this method stems from work developed by Wada and co-workers (further refined by WAVE Life Sciences) and requires a total of seven steps for the preparation of the chiral auxiliary (10, 13-15). Finally, an approach pioneered by Stec and co-workers (Fig. 1B, ii) relies on a similar strategy that begins with a P(III)-oxathiaphospholane loading reaction, followed (again) by oxidative sulfurization to furnish a chiral $\mathrm{P}(\mathrm{V})$-oxathiaphospholane sulfide (OTP) auxiliary; this stable OTP can then be coupled to form the requisite internucleotide linkage using a simple amine activator (typically DBU) (16-18). However, by Stec's own admission, this strategy is scale-limited, suffers poor coupling efficiency, requires resolution of stereorandom OTPs, and is extremely labor intensive (19). Taken together, the deficiencies of current methods point to an overreliance on low valent 
P-O bond forming chemistry, as $\mathrm{P}(\mathrm{III})$ has historically been viewed as the sole gateway for loading (and primary access for coupling) reactions. Reported herein is a major departure from that dogma: the invention of simple, $\mathrm{P}(\mathrm{V})$-based reagents for phosphorus-sulfur incorporation (PSI, stylized as $\psi$, 1) via reactions characterized by exceptional rapidity, scalability, and complete stereocontrol at phosphorus.

Challenging the lore of the field, we opted for an inventive approach with the mission of bypassing P(III) entirely; the reaction sequence should rely exclusively on a $\mathrm{P}(\mathrm{V})$-based platform to forge all key P-heteroatom bonds. This would require the development of a readily accessible reagent with programmable stereochemistry, capable of achieving both loading and coupling steps with robust precision. The realization of this design required a systematic investigation of three key components: (i) an inexpensive chiral backbone to predictably impart stereocontrol, (ii) a safe source of $\mathrm{P}(\mathrm{V})$ to eliminate the previously unavoidable oxidative sulfurization step, and (iii) a reactive yet stable leaving group to enable a facile loading reaction. Ideally, a traceless approach would join the parent species in a direct and asymmetric fashion while liberating a single $\mathrm{P}(\mathrm{S}) \mathrm{O}$ unit with no evidence of the chemical method of installation remaining. These disparate attributes should harmoniously assemble in such a way as to appeal to both process and discovery chemists-traits such as scalability, stability, crystallinity, and rapid reaction times were deemed critical to success. A matrix of candidate backbones, PS sources, and leaving groups was systematically investigated, as summarized in Fig. 1C. The tactical combination of these three constituents proved essential in achievement of the goals set forth above, leading to invention of the $\psi$ reagent system (1, Fig. 1D). Featuring a rigid, limonene-derived backbone anchored to an OTP sulfide core and a stable perfluorinated thiophenol leaving group, this platform embodies an impressive balance of stability and reactivity. The $\psi$ reagents enable the rapid construction of stereopure nucleotide systems and boast an operational simplicity akin to iterative peptide assembly.

Both $(R)$ - and $(S)$-PS linked oligonucleotides can be readily accessed through selection of the desired $\psi$ reagent enantiomer, (+)- $\psi$ and (-)- $\psi$, respectively (Fig. 2A). Derived from limonene, an inexpensive chiral pool terpene and common food additive, these OTP sulfide reagents are produced following epoxidation and ring opening. The loading step refers to the appendage of the first nucleoside to the $\psi$ reagent to deliver a stable OTP-adduct, while the coupling forms the chiral internucleotide linkage. A variety of nucleosides can be loaded in excellent yield (76-96\%, Fig. 2B, 4-7) onto $\psi$ with complete stereocontrol, accompanied by loss of pentafluorothiophenol. When using $\psi$ in excess, no competitive displacement of the OTP-sulfur is observed, despite the fact that this entity is primed for a subsequent coupling step. The 30-min reaction is air and moisture tolerant, occurs at ambient temperature, and requires only DBU as an activator. Remarkably, the next nucleoside in the sequence can be coupled under identical conditions to deliver the dinucleotide as a single diastereoisomer, again in exceptional yield (70-91\%). The intermediate loaded OTP-nucleosides are generally crystalline, bench stable species.

Dimeric combinations of the four native deoxynucleosides, A, T, G, and C, were prepared in high yields with complete stereocontrol; no differences in reaction performance were observed, regardless of base identity (Fig. 2C, 8-17). In a testament to the power of these transformations, both enantiomers of the $\psi$ reagents were made on multigram scale, as were all loaded and coupled compounds. Further, these reactions operate under complete reagent control, as evidenced by the stereochemical outcome in every case: $(+)-\psi$ leads to $(S)$-loaded and $(R)$-coupled products, while $(-)-\psi$ reactions are predictably reversed. In a feature unique to these systems, one can simply invert the coupling sequence order to obtain the opposite P-stereochemistry using the same isomer of $\psi$ reagent (see supplementary materials for a graphical discussion).

Cyclic dinucleotides (CDNs) are macrocyclic natural products endowed with remarkable biological activities and a storied history (25). Ample research indicates that these compounds play a number of prominent biological roles, most notably as secondary messengers in both the mammalian immune system and in bacterial communication (26-31). Importantly, their role as agonists of innate immune response via binding and activation of the stimulator of interferon genes (STING) protein has led to a surge of interest across the pharmaceutical industry $(26,27,32)$. As a consequence, numerous industrial and academic groups have targeted CDN constructs for evaluation as potential therapeutic agents. Just as the PS modification sees a marked improvement in ASO properties, a similar effect has been observed in CDNs, adding a layer of complexity to an already challenging class of natural products $(8,33-40)$. To be sure, the current impediment to rapid clinical progression of these compounds lies in the chemical synthesis. The modular strategies of traditional medicinal chemistry programs are stymied by both the poorly soluble nature of CDNs and the litany of engineered protecting group schemes required to shield the array of heteroatoms found in the nucleobase, sugar core, and P(III)-based starting materials. As shown in Fig. 3A, nine steps are generally required from the parent nucleoside to prepare a single CDN as a mixture of all four possible diastereoisomers, with an observed ratio that appears to be substrate dependent (33-40). Of this arduous sequence, only four steps can be considered construction reactions, or those that contribute directly to $\mathrm{P}-\mathrm{O}$ bond formation. The remainder account for various synthetic concessions, including protecting 
group manipulations and $\mathrm{P}(\mathrm{III})$ to $\mathrm{P}(\mathrm{V})$ oxidations. As compared to ASOs, the challenge of incorporating chiral PS linkages into CDNs becomes far more profound, as one is at the mercy of substrate bias to obtain diastereoselectivity at phosphorus. Modular, stereocontrolled CDN synthesis has so far proven elusive (Fig. 3A). In contrast, $\psi$ reagents enable a dramatic divergence from the traditional P(III) approach, requiring only 4 to 5 steps from a starting nucleoside to arrive at a stereopure CDN. Two general protocols for CDN synthesis have been devised, as outlined in Fig. 3B. The stepwise approach ensures complete transfer of stereochemical information from the $\psi$ reagent by forging the CDN macrocycle one $\mathrm{P}-\mathrm{O}$ bond at a time. To this point, CDN 21 was constructed as a single diastereoisomer in $14 \%$ overall yield via an operationally simple sequence: first, the desired $\psi$ reagent is loaded onto a nucleoside at either the 3 ' or 5 ' -position, then coupled to a resin-bound nucleoside (itself anchored to the solid-support). The dinucleotide is then cleaved from the solid-support to reveal the remaining free alcohol with no purification required. The final coupling reaction is achieved within ten minutes to afford the desired CDN target. Conversely, the concerted protocol accomplishes macrocyclization directly from the linear dinucleotide diol, as in $\mathbf{2 0}$. Notably, CDN 21, procured in stereopure form using the stepwise approach, was accessed in $44 \%$ yield via the concerted method, albeit with 3:1 diastereomeric ratio (d.r.), a result of the aforementioned competitive $3^{\prime}$ versus $5^{\prime} \psi$ loading in the penultimate bond formation (Fig. 3C, Path A versus Path B). Though step-economic, the concerted sequence only effects high d.r. when the reactivity of the $3^{\prime}$ and $5^{\prime}$ alcohols is sufficiently differentiated to enable selective reactivity with the $\psi$ reagent. As with the synthesis of linear dinucleotides (Fig. $2 \mathrm{C}$ ), the order of assembly can be used to program the resulting phosphorus stereochemistry; thus, $\mathbf{1 8}$ was synthesized as three distinct stereoisomers, each with perfect stereocontrol, while CDN 19 was obtained in $24 \%$ overall yield as a single diastereoisomer. Both approaches can be conducted both in solution and on solid-phase with similar facility.

Beyond dimeric nucleotides, fascination surrounding $\mathrm{P}(\mathrm{III})$ in the context of general solid-phase oligonucleotide construction persists. This has resulted in a staggering number of publications and an entire industry of specialized equipment built to accommodate the complex reactivity and sensitivity of P(III)-derived systems. Perhaps unsurprisingly, reports of $\mathrm{P}(\mathrm{V})$ approaches to solid-phase oligonucleotide synthesis (SPOS) of PS-analogs remain conspicuously absent. To this end, an unoptimized automated load-deprotect-couple $\mathrm{P}(\mathrm{V})$-cycle (Fig. 4) was employed, using equipment designed for P(III)-chemistry, to furnish the homo-dT pentamer (23) as a single diastereoisomer in $23 \%$ yield. Notably, $\mathbf{2 3}$ was constructed without rigorous exclusion of air and moisture, in stark contrast to the operational constraints of traditional
P(III)-SPOS. Under traditional automated phosphoramidite conditions, the same oligonucleotide was obtained as a mixture of 16 diastereoisomers in $63 \%$ yield. In a promising demonstration, the 16-mer $\mathbf{2 4}$ was successfully constructed using the unoptimized $\psi$ approach, though with an expected drop in crude purity (see supplementary materials for details). Indeed, the introduction of $\psi$ reagents engenders an advantageous approach to oligonucleotide synthesis enabled by a simple, reagent-based strategy capable of similarly effective solution- and solid-phase chemistry.

\section{REFERENCES AND NOTES}

1. C. E. Dunbar, K. A. High, J. K. Joung, D. B. Kohn, K. Ozawa, M. Sadelain, Gene therapy comes of age. Science 359, eaan4672 (2018). doi:10.1126/science.aan4672 Medline

2. E. W. Ottesen, ISS-N1 makes the first FDA-approved drug for spinal muscular atrophy. Transl. Neurosci. 8, 1-6 (2017). doi:10.1515/tnsci-2017-0001Medline

3. H. P. Vosberg, F. Eckstein, Effect of deoxynucleoside phosphorothioates incorporated in DNA on cleavage by restriction enzymes. J. Biol. Chem. 257, 6595-6599 (1982). Medline

4. S. T. Cooke, Antisense Research and Applications, $1^{\text {st }}$ ed. (Springer-Verlag Berlin Heidelberg, New York, 1998)

5. E. Wickstrom, Oligodeoxynucleotide stability in subcellular extracts and culture media. J. Biochem. Biophys. Methods 13, 97-102 (1986). doi:10.1016/0165$022 \times(86) 90021-7$ Medline

6. J. Purcell, A. C. Hengge, The thermodynamics of phosphate versus phosphorothioate ester hydrolysis. J. Org. Chem. 70, 8437-8442 (2005). doi:10.1021/j00511997 Medline

7. C. A. Stein, D. Castanotto, FDA-approved oligonucleotide therapies in 2017. Mol Ther. 25, 1069-1075 (2017). doi:10.1016/j.ymthe.2017.03.023 Medline

8. J. Li, M. D. Eastgate, Current complexity: A tool for assessing the complexity of organic molecules. Org. Biomol. Chem. 13, 7164-7176 (2015). doi:10.1039/C50B00709G Medline

9. H. G. Bohr, I. Shim, C. Stein, H. Ørum, H. F. Hansen, T. Koch, Electronic structures of LNA phosphorothioate oligonucleotides. Mol. Ther. Nucleic Acids 8, 428-441 (2017). doi:10.1016/j.omtn.2017.05.011 Medline

10. N. Iwamoto, D. C. D. Butler, N. Svrzikapa, S. Mohapatra, I. Zlatev, D. W. Y. Sah, S M. Meena, S. M. Standley, G. Lu, L. H. Apponi, M. Frank-Kamenetsky, J. J. Zhang, C. Vargeese, G. L. Verdine, Control of phosphorothioate stereochemistry substantially increases the efficacy of antisense oligonucleotides. Nat. Biotechnol. 35, 845-851 (2017). doi:10.1038/nbt.3948 Medline

11. S. L. Beaucage, M. H. Caruthers, Deoxynucleoside phosphoramidites-A new class of key intermediates for deoxypolynucleotide synthesis. Tetrahedron Lett. 22 , 1859-1862 (1981). doi:10.1016/S0040-4039(01)90461-7

12. M. H. Caruthers, Synthesis and Applications of DNA and RNA, ed. S. A. Narang, (Academic Press, Orlando, 1987), pp. 47-94

13. N. Oka, M. Yamamoto, T. Sato, T. Wada, Solid-phase synthesis of stereoregular oligodeoxyribonucleoside phosphorothioates using bicyclic oxazaphospholidine derivatives as monomer units. J. Am. Chem. Soc. 130, 16031-16037 (2008) doi:10.1021/ja805780u Medline

14. Y. Nukaga, K. Yamada, T. Ogata, N. Oka, T. Wada, Stereocontrolled solid-phase synthesis of phosphorothioate oligoribonucleotides using 2'-0-(2cyanoethoxymethyl)-nucleoside 3'-0-oxazaphospholidine monomers. J. Org. Chem. 77, 7913-7922 (2012). doi:10.1021/jo301052v Medline

15. N. Oka, T. Kondo, S. Fujiwara, Y. Maizuru, T. Wada, Stereocontrolled synthesis of oligoribonucleoside phosphorothioates by an oxazaphospholidine approach. Org. Lett. 11, 967-970 (2009). doi:10.1021/ol802910k Medline

16. W. J. Stec, A. Grajkowski, M. Koziolkiewicz, B. Uznanski, Novel route to oligo(deoxyribonucleoside phosphorothioates). Stereocontrolled synthesis of Pchiral oligo(deoxyribonucleoside phosphorothioates). Nucleic Acids Res. 19 5883-5888 (1991). doi:10.1093/nar/19.21.5883 Medline

17. W. J. Stec, A. Grajkowski, A. Kobylanska, B. Karwowski, M. Koziolkiewicz, K 
Misiura, A. Okruszek, A. Wilk, P. Guga, M. Boczkowska, Diastereomers of nucleoside 3'-0-(2-thio-1,3,2-oxathia(selena)phospholanes): Building blocks for stereocontrolled synthesis of oligo(nucleoside phosphorothioate)s. J. Am. Chem. Soc. 117, 12019-12029 (1995). doi:10.1021/ja00154a001

18. W. J. Stec, B. Karwowski, M. Boczkowska, P. Guga, M. Koziołkiewicz, M. Sochacki, M. W. Wieczorek, J. Błaszczyk, Deoxyribonucleoside 3'-0-(2-thio- and 2-oxospiro-4,4-pentamethylene-1,3,2-oxathiaphospholane)s: Monomers for stereocontrolled synthesis of oligo(deoxyribonucleoside phosphorothioate)s and chimeric PS/PO oligonucleotides. J. Am. Chem. Soc. 120, 7156-7167 (1998). doi:10.1021/ia973801j

19. P. Guga, W. J. Stec, Synthesis of phosphorothioate oligonucleotides with stereodefined phosphorothioate linkages. Curr. Protoc. Nucleic Acid Chem. 14, 4.17.1-4.17.28 (2003). doi:10.1002/0471142700.nc0417s14 Medline

20. N. Iwamoto, N. Oka, T. Sato, T. Wada, Stereocontrolled solid-phase synthesis of oligonucleoside $\mathrm{H}$-phosphonates by an oxazaphospholidine approach. Angew. Chem. Int. Ed. 48, 496-499 (2009). doi:10.1002/anie.200804408 Medline

21. R. P. Iyer, D. Yu, N.-H. Ho, W. Tan, S. Agrawal, A novel nucleotide phosphoramidite synthon derived from $1 R$, 2S-ephedrine. Tetrahedron Asymmetry 6, 1051-1054 (1995). doi:10.1016/0957-4166(95)00122-6

22. M. Guo, D. Yu, R. P. Iyer, S. Agrawal, Solid-phase stereoselective synthesis of 2'O-methyl-oligoribonucleoside phosphorothioates using nucleoside bicyclic oxazaphospholidines. Bioorg. Med. Chem. Lett. 8, 2539-2544 (1998). doi:10.1016/S0960-894X(98)00450-8 Medline

23. A. Wilk, A. Grajkowski, L. R. Phillips, S. L. Beaucage, Deoxyribonucleoside cyclic Nacylphosphoramidites as a new class of monomers for the stereocontrolled synthesis of oligothymidylyl- and oligodeoxycytidylyl-phosphorothioates. J. Am. Chem. Soc. 122, 2149-2156 (2000). doi:10.1021/ja991773u

24. M. Li, H. L. Lightfoot, F. Halloy, A. L. Malinowska, C. Berk, A. Behera, D. Schümperli, J. Hall, Synthesis and cellular activity of stereochemically-pure 2'-0-(2methoxyethyl)-phosphorothioate oligonucleotides. Chem. Commun. (Camb.) 53 , 541-544 (2017). doi:10.1039/C6CC08473G Medline

25. P. Clivio, S. Coantic-Castex, D. Guillaume, (3'-5')-Cyclic dinucleotides: Synthetic strategies and biological potential. Chem. Rev. 113, 7354-7401 (2013). doi:10.1021/cr300011s Medline

26. D. L. Burdette, K. M. Monroe, K. Sotelo-Troha, J. S. Iwig, B. Eckert, M. Hyodo, Y. Hayakawa, R. E. Vance, STING is a direct innate immune sensor of cyclic di-GMP. Nature 478, 515-518 (2011). doi:10.1038/nature10429 Medline

27. L. Sun, J. Wu, F. Du, X. Chen, Z. J. Chen, Cyclic GMP-AMP synthase is a cytosolic DNA sensor that activates the type I interferon pathway. Science 339, 786-791 (2013). doi:10.1126/science.1232458 Medline

28. M. Gomelsky, cAMP, c-di-GMP, c-di-AMP and now cGMP: Bacteria use them all. Mol. Microbiol. 79, 562-565 (2011). doi:10.1111/j.1365-2958.2010.07514.x Medline

29. P. Ross, Y. Aloni, C. Weinhouse, D. Michaeli, P. Weinberger-Ohana, R. Meyer, M. Benziman, An unusual guanyl oligonucleotide regulates cellulose synthesis in Acetobacter xylinum. FEBS Lett. 186, 191-196 (1985). doi:10.1016/00145793(85)80706-7 Medline

30. P. Ross, Y. Aloni, H. Weinhouse, D. Michaeli, P. Weinberger-Ohana, R. Mayer, M. Benziman, Control of cellulose synthesis Acetobacter xylinum. A unique guanyl oligonucleotide is the immediate activator of the cellulose synthase. Carbohydr. Res. 149, 101-117 (1986). doi:10.1016/S0008-6215(00)90372-0

31. P. Ross, H. Weinhouse, Y. Aloni, D. Michaeli, P. Weinberger-Ohana, R. Mayer, S. Braun, E. de Vroom, G. A. van der Marel, J. H. van Boom, M. Benziman, Regulation of cellulose synthesis in Acetobacter xylinum by cyclic diguanylic acid. Nature 325, 279-281 (1987). doi:10:1038/325279a0 Medline

32. R. Cross, STING fever is sweeping through the cancer immunotherapy world. Chem. Eng. News 96, 24-26 (2018).

33. C. Battistini, S. Fustinoni, M. G. Brasca, D. Borghi, Stereoselective synthesis of cyclic dinucleotide phosphorothioates. Tetrahedron 49, 1115-1132 (1993). doi:10.1016/S0040-4020(01)86292-X

34. P. Guga, B. Karwowski, D. Błaziak, M. Janicka, A. Okruszek, B. Rębowska, W. J. Stec, Cyclization versus oligomerization of $\mathrm{SP}_{\mathrm{P}}$ and $\mathrm{RP}-5^{\prime}-\mathrm{OH}-\mathrm{N}^{4}$-benzoyl-2'deoxycytidine-3'-0-(2-thio-4,4-pentamethylene-1,3,2-oxathiaphospholane)s. Tetrahedron 62, 2698-2704 (2006). doi:10.1016/j.tet.2005.12.022

35. H. Yan, A. L. Aguilar, Synthesis of 3',5'-cyclic diguanylic acid (cdiGMP) using 1-(4chlorophenyl)-4-ethoxypiperidin-4-yl as a protecting group for 2'-hydroxy functions of ribonucleosides. Nucleosides Nucleotides Nucleic Acids 26, 189-204 (2007). doi:10.1080/15257770601112762 Medline

36. H. Yan, X. Wang, R. KuoLee, W. Chen, Synthesis and immunostimulatory properties of the phosphorothioate analogues of cdiGMP. Bioorg. Med. Chem. Lett. 18, 5631-5634 (2008). doi:10.1016/j.bmcl.2008.08.088 Medline

37. J. Zhao, E. Veliath, S. Kim, B. L. Gaffney, R. A. Jones, Thiophosphate analogs of cdi-GMP: Impact on polymorphism. Nucleosides Nucleotides Nucleic Acids 28 , 352-378 (2009). doi:10.1080/15257770903044523 Medline

38. B. L. Gaffney, E. Veliath, J. Zhao, R. A. Jones, One-flask syntheses of c-di-GMP and the $\left[R_{\mathrm{p}}, R_{\mathrm{p}}\right]$ and $\left[R_{\mathrm{p}}, S_{\mathrm{p}}\right]$ thiophosphate analogues. Org. Lett. 12, 3269-3271(2010). doi:10.1021/ol101236b Medline

39. N. Fei, D. Häussinger, S. Blümli, B.-J. Laventie, L. D. Bizzini, K. Zimmermann, U. Jenal, D. Gillingham, Catalytic carbene transfer allows the direct customization of cyclic purine dinucleotides. Chem. Commun. (Camb.) 50, 8499-8502 (2014). doi:10.1039/C4CC01919A Medline

40. T. Lioux, M.-A. Mauny, A. Lamoureux, N. Bascoul, M. Hays, F. Vernejoul, A.-S. Baudru, C. Boularan, J. Lopes-Vicente, G. Qushair, G. Tiraby, Design, synthesis, and biological evaluation of novel cyclic adenosine-inosine monophosphate (CAIMP) analogs that activate stimulator of interferon genes (STING). J. Med. Chem. 59, 10253-10267 (2016). doi:10.1021/acs. medchem.6b01300 Medline

41. D. Steiner, L. Ivison, C. T. Goralski, R. B. Appell, J. R. Gojkovic, B. Singaram, A facile and efficient method for the kinetic separation of commercially available cis- and trans-limonene epoxide. Tetrahedron Asymmetry 13, 2359-2363 (2002). doi:10.1016/S0957-4166(02)00646-8

42. H. Hachiya et al., Unique salt effect on the high yield synthesis of acid-labile terpene oxides using hydrogen peroxide under acidic aqueous conditions. Synlett 19, 2819-2822 (2011)

43. Y. Nagaya, Y. Kitamura, R. Nakashima, A. Shibata, M. Ikeda, Y. Kitade, Practical and reliable synthesis of 1,2-dideoxy-d-ribofuranose and its application in RNA studies. Nucleosides Nucleotides Nucleic Acids 35, 64-75 (2016). doi:10.1080/15257770.2015.1114128 Medline

44. H. Huang, R. S. Das, A. K. Basu, M. P. Stone, Structure of (5'S)-8,5'-cyclo-2'deoxyguanosine in DNA. J. Am. Chem. Soc. 133, 20357-20368 (2011) doi:10.1021/ja207407n Medline

45. D. Hutter, M.-J. Kim, N. Karalkar, N. A. Leal, F. Chen, E. Guggenheim, V. Visalakshi, J. Olejnik, S. Gordon, S. A. Benner, Labeled nucleoside triphosphates with reversibly terminating aminoalkoxyl groups. Nucleosides Nucleotides Nucleic Acids 29, 879-895 (2010). doi:10.1080/15257770.2010.536191 Medline

46. K. S. Krishnakumar, P. Strazewski, Synthesis of a deoxyxylopuromycin analogue. Synlett 7, 1055-1058 (2010).

47. S. F. Wnuk, D. R. Companioni, V. Neschadimenko, M. J. Robins, The $\beta$-fluorine effect. Electronic versus steric effects in radical deoxygenations of fluorinecontaining pentofuranose nucleosides. J. Org. Chem. 67, 8794-8797 (2002). doi:10.1021/j0020428b Medline

48. R. Gao, C. D. Claeboe, B. M. Eisenhauer, S. M. Hecht, Identification of specific nonbridging phosphate oxygens important for DNA cleavage by human topoisomerase I. Biochemistry 43, 6167-6181 (2004). doi:10.1021/bi040005z Medline

\section{ACKNOWLEDGMENTS}

We are grateful to Dr. Brian Fink and Dr. Artiom Cernijenko for insightful discussions; Dr. Jason Chen (TSRI) for assistance with analysis; and Dr. D.-H. Huang and Dr. L. Pasternack (TSRI) for assistance with nuclear magnetic resonance spectroscopy. Professor A. L. Rheingold, Dr. C. E. Moore, and Dr. Milan Gembicky (UCSD) are acknowledged for x-ray analysis. Funding: Financial support for this work was provided by NIH (grant number GM-118176), BristolMyers Squibb, NSF GRFP (J.N.D.). Author contributions: K.W.K., J.N.D., M.A.S. M.D.E., and P.S.B. conceived of the work. K.W.K., J.N.D., M.A.S., B.Z., J.C.V., C.K., S.E.M., I.M.M., Y.Z., C.H., J.Z., C.Y., and Q.W. designed and executed all experiments and performed all data analysis. R.E.O. and P.P. provided specific insight and direction for the ASO and CDN studies, respectively. K.W.K., J.N.D., and P.S.B. wrote the manuscript; M.A.S., M.D.E., R.E.O., and I.M.M. assisted with editing. Competing interests: A provisional U.S. patent application on this work has been filed (application number 62657551), with K.W.K, J.N.D., M.A.S., B.Z., 
M.D.E., I.M.M., R.E.O., and P.S.B. listed as inventors. P.S.B. is a paid consultant for Bristol-Myers Squibb. Data and materials availability: Crystallographic data are available free of charge from the Cambridge Crystallographic Data Centre under reference CCDC 1840250, 1849772 and 1849773. Experimental procedures, frequently asked questions, extensive optimization data, ${ }^{1} \mathrm{H}$ NMR spectra, ${ }^{13} \mathrm{C}$ NMR spectra, ${ }^{31} \mathrm{P}$ NMR spectra, analytical HPLC traces, and MS data are available in the supplementary materials.

\section{SUPPLEMENTARY MATERIALS}

www. sciencemag.org/cgi/content/full/science.aau3369/DC1

Materials and Methods

Supplementary Text

Figs. S1 to S4

Tables S1 to S5

References (41-48)

NMR Spectra

4 June 2018; accepted 12 July 2018

Published online 2 August 2018

10.1126/science.aau3369 
A Antisense Oligonucleotides: A Monumental Stereochemical Challenge

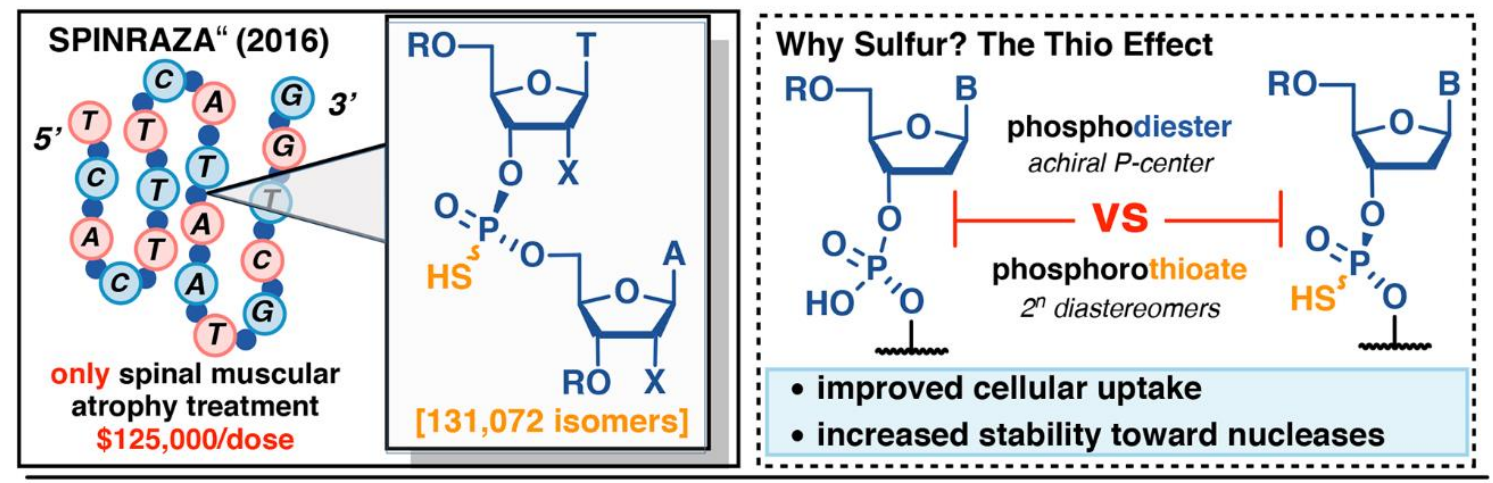

B The P(III) Scaffold: Primary Gateway to Phosphorothioates

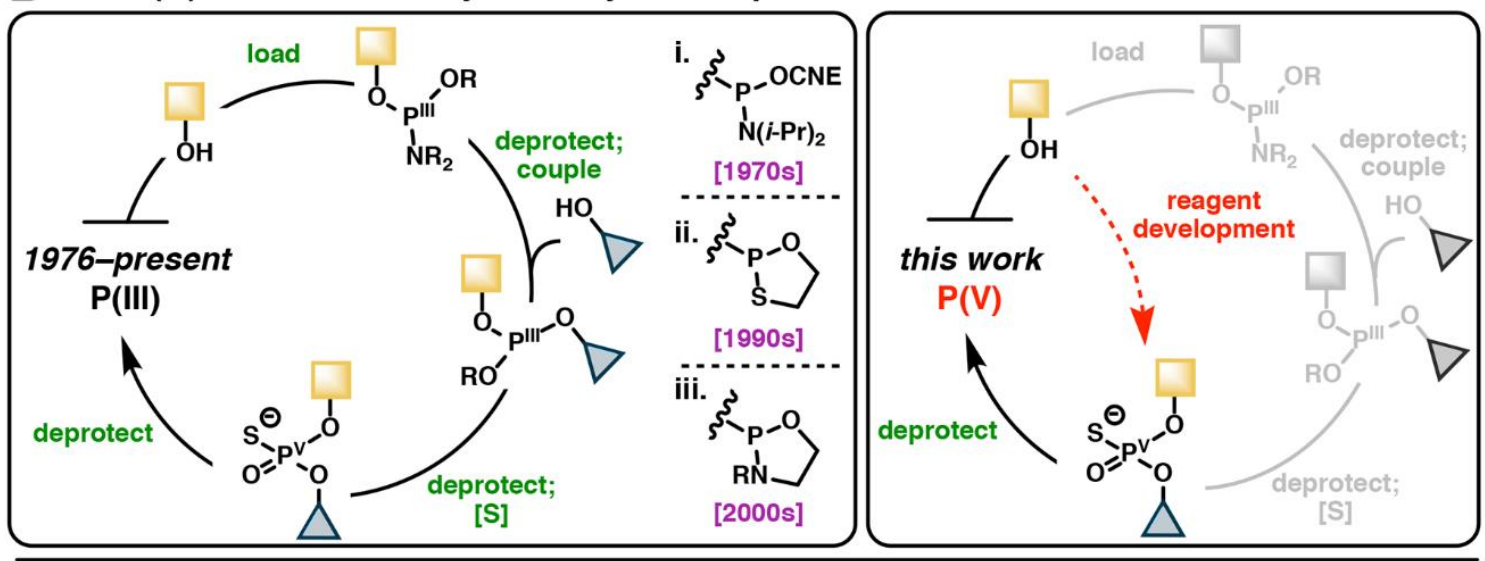

C Bypassing P(III): The Quest to Develop an Ideal P(V) Reagent

[3 key components]

no chromatography required

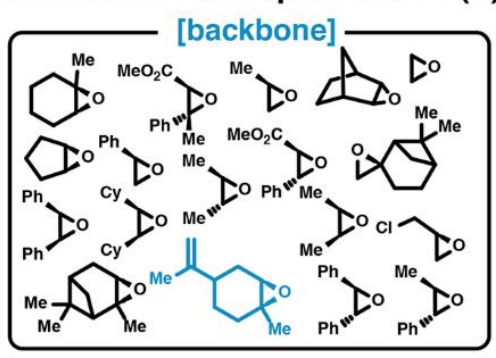

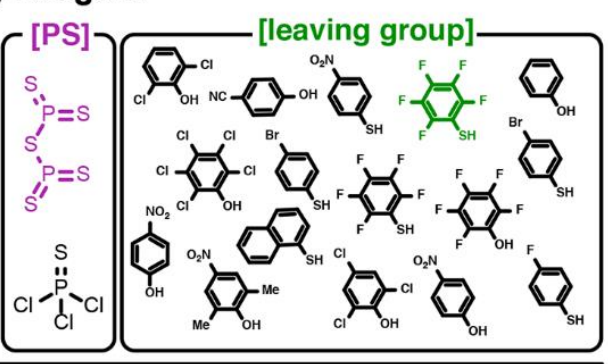

D Psi Reagent: A Simple, Redox-Economic Alternative<smiles>C=C(C)[C@H]1CC[C@@]2(C)O[C@H]2C1</smiles>

(-)-limonene oxide (2) [chiral backbone]<smiles>CCCCSP(=S)(S)SN(CC)CC</smiles>

1 step (3)

$[\mathrm{P}(\mathrm{V})-\mathrm{S}$ source $]$<smiles>C=C(C)[C@H]1CC[C@@](C)(SP(=S)([S-])C(F)(F)F)[C@H](O)C1</smiles>

PSI Reagent (1)

[phosphorus-sulfur incorporation, $\psi$ ]
- stereoselective

• redox-economic

• simplified protocol

- solid-phase

- solution-phase

$\bullet$ rapid

- scale amenable

Fig. 1. Background and development. (A) Inspiration, (B) historical context, (C) development, (D) invention of the $\psi$ reagent platform. 
A Synthesis and stereochemistry of $\psi$ reagents

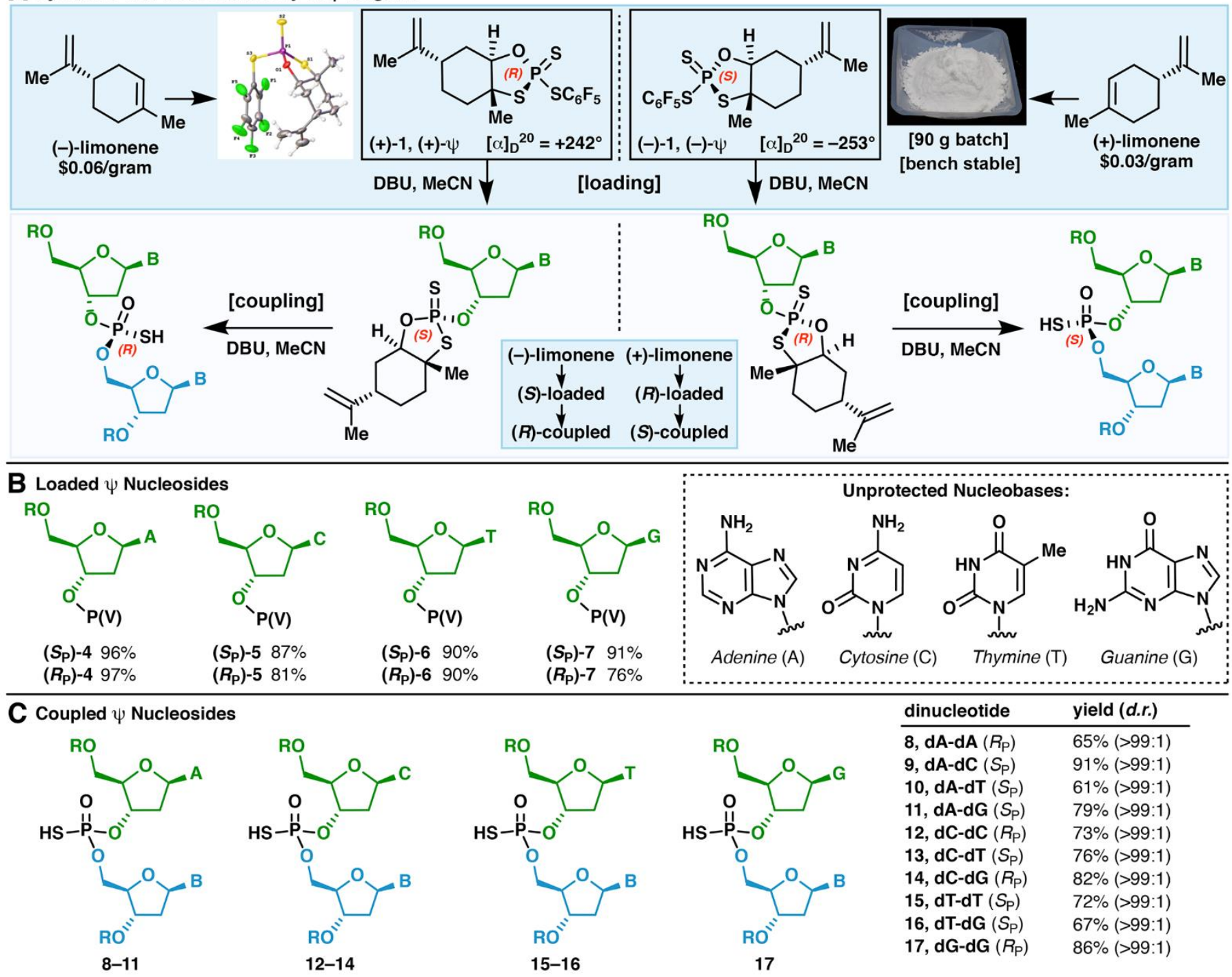

Fig. 2. Formalisms for the use of $\psi$ reagents in dinucleotide synthesis. (A) Stereochemical assignments, (B) loading of nucleoside monomers, $(\mathrm{C})$ coupling to produce stereopure phosphorothioate dinucleotides. $\mathrm{R}=$ TBDPS. Loading: nucleoside (1 equiv.), $\psi$ reagent (1.3 equiv.), $\mathrm{DBU}$ (1.3 equiv.), $\mathrm{MeCN}, 25^{\circ} \mathrm{C}, 30$ min. Coupling: nucleoside-P( $\mathrm{V})\left(1.0\right.$ equiv.), coupling partner (2.0 equiv.), $\mathrm{DBU}$ (3.0 equiv.), $\mathrm{MeCN}, 25^{\circ} \mathrm{C}, 30 \mathrm{~min}$. 


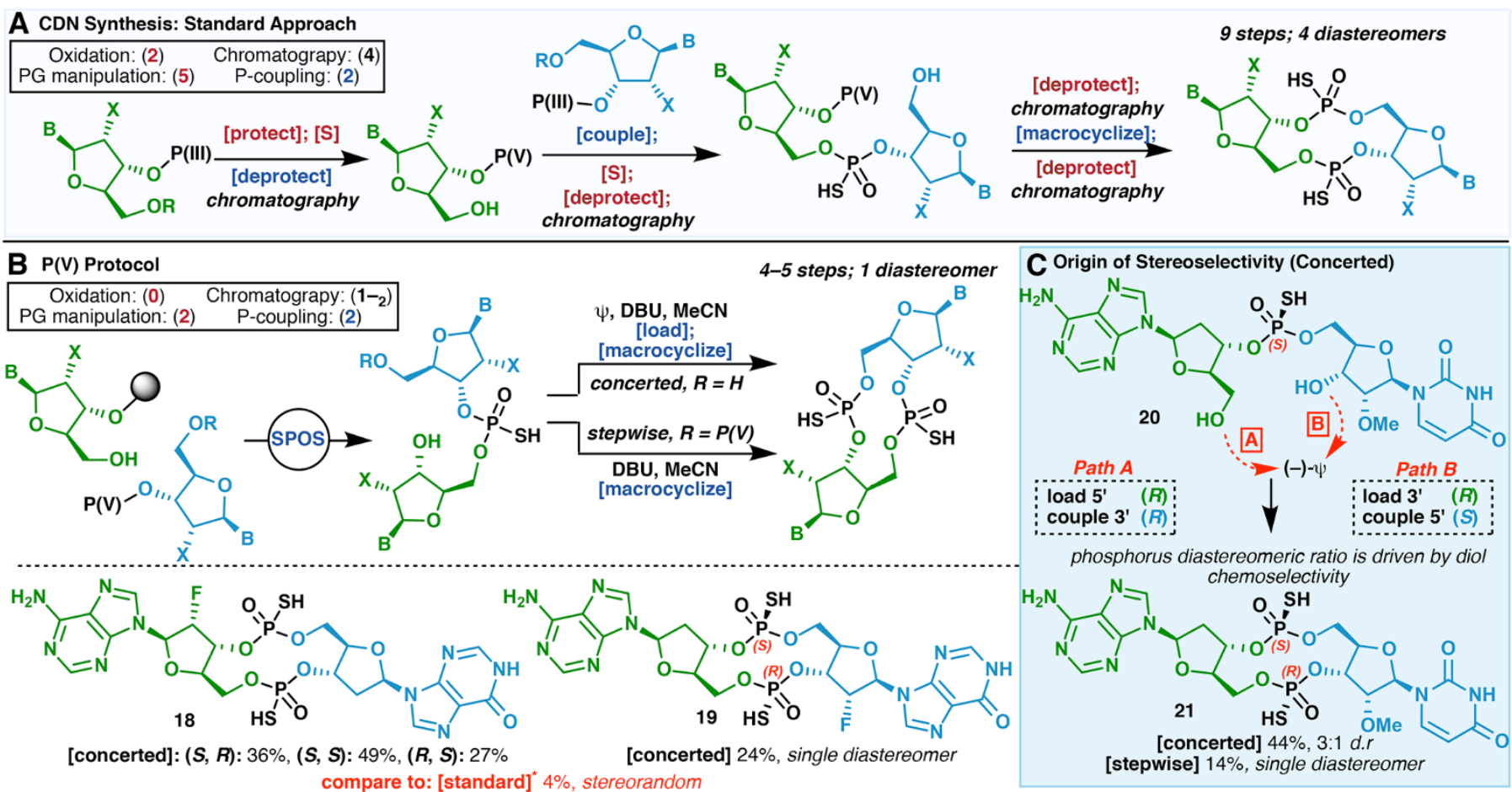

Fig. 3. Applications of the $\psi$-reagent platform to the simplified synthesis of CDNs. (A) Prior art, (B) stepwise and concerted macrocyclization using $\psi,(C)$ origin of observed stereochemistry in concerted protocol. ${ }^{*}$ See reference (40) for the literature method. 


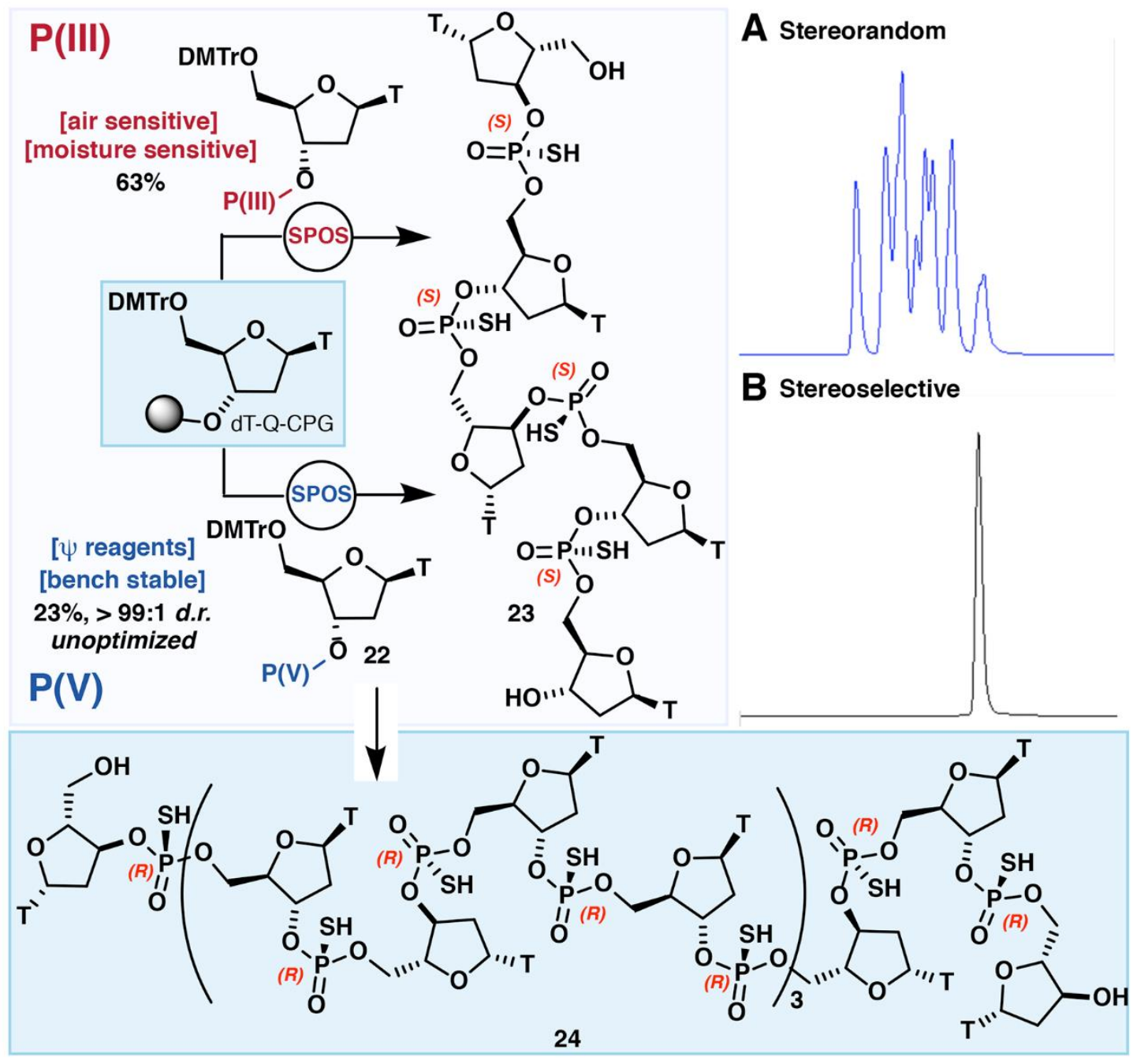

Fig. 4. Automated synthesis of PS-oligonucleotides. (A) Crude HPLC trace of pentamer 23 (16 diastereoisomers) synthesized under standard P(III) automated conditions, (B) crude HPLC trace of pentamer 23 (1 diastereoisomer) synthesized under unoptimized $\psi$ automated conditions. 